\title{
Comparison of the performance of five screening methods for sarcopenia
}

This article was published in the following Dove Press journal:

Clinical Epidemiology

\author{
Médéa Locquet' \\ Charlotte Beaudart' \\ Jean-Yves Reginster' \\ Jean Petermans ${ }^{2}$ \\ Olivier Bruyère' \\ 'Department of Public Health, \\ Epidemiology and Health Economics, \\ WHO Collaborating Centre for Public \\ Health Aspects of Musculoskeletal \\ Health and Aging, University of Liège, \\ ${ }^{2}$ Geriatric Department, $\mathrm{CHU}$ of Liège, \\ Liège, Belgium
}

Correspondence: Médéa Locquet Department of Public Health, Epidemiology and Health Economics, WHO Collaborating Centre for Public Health Aspects of Musculoskeletal Health and Aging, University of Liège, CHU Bât B.23, Avenue Hippocrate,

13, 4000 Liège, Belgium

Tel +32 43662519

Email medea.locquet@ulg.ac.be
Background: Sarcopenia leads to serious adverse health consequences. There is a dearth of screening tools for this condition, and performances of these instruments have rarely been evaluated. Our aim was to compare the performance of five screening tools for identifying elders at risk of sarcopenia against five diagnostic definitions.

Subjects and methods: We gathered cross-sectional data of elders from the SarcoPhAge ("Sarco"penia and "Ph"ysical Impairment with Advancing "Age") study. Lean mass was measured with X-ray absorptiometry, muscle strength with a dynamometer and physical performance with the Short Physical Performance Battery (SPPB) test. Performances of screening methods were described using sensitivity, specificity, positive predictive value (PPV), negative predictive value (NPV) and area under the curve (AUC), according to five diagnostic definitions of sarcopenia. For each screening tool, optimal cutoff points were computed using two methods. Results: A total of 306 subjects ( $74.8 \pm 5.9$ years, $59.5 \%$ women) were included. The prevalence of sarcopenia varied from $5.7 \%$ to $16.7 \%$ depending on the definition. The best sensitivity (up to $100 \%$ ) and the best NPV (up to $99.1 \%$ ) were obtained with the screening test of Ishii et al, regardless of the definition applied. The highest AUC (up to 0.914) was also demonstrated by the instrument of Ishii et al. The most specific tool was the algorithm of the European Working Group on Sarcopenia in Older People (EWGSOP; up to 91.1\%). All NPVs were above 87.0\%, and all PPVs were below 51.0\%. New cutoffs related to each screening instrument were also proposed to better discriminate sarcopenic individuals from non-sarcopenic individuals.

Conclusion: Screening instruments for sarcopenia can be relevantly used in clinical practice to make sure to identify individuals who do not suffer from the syndrome. The screening test of Ishii et al showed better properties in terms of distinguishing those at risk of sarcopenia from those who were not at risk.

Keywords: sarcopenia, screening, older individuals, sensitivity, specificity, ROC analysis

\section{Introduction}

Advancing age is synonymous with substantial changes in body composition. Indeed, lean mass decreases after the age of 50 years to reach a loss of $15 \%$ per decade at approximately 70 years. ${ }^{1}$ Moreover, a faster decrease in muscle quality (i.e., muscle strength and ability) is observed. ${ }^{2}$ This relationship between aging and loss of muscle function is now well documented and better studied. ${ }^{3}$ When the muscular decline is too important, it is referred to as "sarcopenia," a condition that causes significant adverse consequences in terms of not only functional disabilities, fractures, hospitalizations and increased mortality ${ }^{4}$ but also quality of life $^{5}$ and health care expenditure allocable to the pathology. ${ }^{6}$ The importance of sarcopenia in terms of public health burden is 
also given by the current prevalence of the affection, ${ }^{7}$ which is expected to grow significantly (i.e., the prevalence rate could increase by $72.4 \%$ in 2045 in subjects aged 65 years and older). ${ }^{8}$

However, even if it is now well acknowledged that sarcopenia reflects a generalized loss of muscle mass accompanied by decline in muscular strength and function, ${ }^{9}$ the scientific community is still faced with a debate regarding optimal cutoff values and diagnostic criteria to apply. The variety of thresholds proposed implies considerable repercussions in the characterization and management of the condition. ${ }^{10-12}$ In addition, the specialized equipment for diagnosing sarcopenia in clinical practice has some significant limitations. Indeed, the currently proposed techniques for measuring muscle mass, such as bioelectrical impedance analysis (BIA) and dual-energy X-ray absorptiometry (DEXA), ${ }^{13}$ present a non-negligible cost and availability problems and are time-consuming, while others, such as magnetic resonance imaging (MRI) or computed tomography (CT), have the further disadvantage of exposing individuals (patients and practitioners) to significant radiation.

Therefore, to limit the use of these resource-consuming diagnostic devices, some study teams have tried to develop a preliminary step by creating screening methods, thus helping the clinicians to determine whether it is necessary to resort to a more demanding diagnostic instrument to objectify the presence of sarcopenia. Indeed, the early identification of older adults likely to suffer from sarcopenia would allow them to implement, at an early stage, preventive strategies ${ }^{14}$ to avoid severe health events (e.g., fractures and hospitalization), which appear once the disease is apparent and well settled. We have identified five screening strategies for the detection of subjects at risk of sarcopenia, allowing us to determine whether a more sophisticated diagnostic assessment procedure is necessary or not. A former study ${ }^{15}$ integrated the main characteristics of these five methods as well as their respective strengths and limits. However, no comparison of their performances has yet been performed within the same population set. Based on data from the SarcoPhAge (Sarcopenia and Physical Impairment with Advancing Age) cohort, a Belgian cohort study, our aim was to perform a head-to-head comparison of the performances of the existing screening tools to determine which is the most effective in predicting elders at risk of sarcopenia and to verify their accuracy and clinical relevance by challenging them against different definitions of the clinical diagnosis of sarcopenia.

\section{Subjects and methods Description and participant population of the SarcoPhAge study}

To perform the current analysis, we gathered cross-sectional data of older individuals from the SarcoPhAge study, an ongoing prospective study seeking to collect substantial scientific data characterizing sarcopenia in a population of older subjects. This project started in 2013 and monitors participants every year, with a main objective of assessing several health and functional consequences of sarcopenia. The subjects of the study are healthy individuals who are aged 65 years and older and who were recruited in different departments of an outpatient clinic in Liège, Belgium, and through press advertisements. The methods used and baseline results have been previously described. ${ }^{5}$ Each participant was required to learn about the aims of the study and to sign an informed consent form. The research protocol and its amendments were reviewed and approved by the Ethics Committee of the University Teaching Hospital of Liège under the reference 2012-277.

For this ancillary analysis, 336 older individuals from the SarcoPhAge study were involved, consisting of the population still present in the study after the second year of follow-up (i.e., all subjects reviewed between July 2015 and July 2016). Because some have not completed the entire examinations and questionnaires $(n=30)$, the data of 306 subjects were available and analyzed for the current investigation.

\section{Diagnosis of sarcopenia: evaluation and definitions}

The diagnosis of sarcopenia was based upon the measurement of three main components:

- An assessment of muscle mass: the skeletal muscle index (SMI) was recorded using DEXA (Hologic Discovery A, Marlborough, MA, USA), calibrated daily by scanning a spine phantom in accordance with the manufacturer's instructions. The SMI was calculated as appendicular skeletal lean mass (ALM; i.e., the sum of the muscle mass in both arms and legs) divided by height squared.

- An assessment of muscle strength: the handgrip strength was evaluated by a handheld dynamometer (Saehan Corporation, Kyungsangnam-Do, Republic of Korea), calibrated each year for 10, 40 and $90 \mathrm{~kg}$. Participants had to squeeze the device as hard as they can three times in each hand. We recorded the highest of the six values. ${ }^{16}$

- An evaluation of physical ability: the Short Physical Performance Battery (SPPB) test ${ }^{17}$ was used to assess 
physical performance. It consisted of three separate tests: balance, $4 \mathrm{~m}$ gait speed and chair stand test. A score between 0 and 4 was assigned for each component (with a maximum of 12 points).

To determine the accuracy of our findings in this current study and their clinical relevance, we decided to perform an analysis across several diagnostic definitions of sarcopenia. Five main diagnostic classifications are now widely used in sarcopenia study and are the results of various working groups: Cruz-Jentoft et $\mathrm{al}^{18}$ for the European Working Group on Sarcopenia in Older People (EWGSOP); Fielding et $\mathrm{al}^{19}$ for the International Working Group on Sarcopenia (IWGS); Morley et $\mathrm{al}^{20}$ for the Society of Sarcopenia, Cachexia and Wasting Disorders; Chen et $\mathrm{al}^{21}$ for the Asian Working Group for Sarcopenia (AWGS) and Studenski et $\mathrm{al}^{22}$ for the Foundation for the National Institutes of Health Biomarkers Consortium (FNHI) Sarcopenia Project.

To highlight a weakness of muscle mass, the definitions of Cruz-Jentoft et al, Fielding et al, Morley et al and Chen et al consider sex-specific thresholds based on the SMI (Table 1). The classification of Studenski et al uses specific thresholds of the $\mathrm{ALM}_{\mathrm{BMI}}$ for men and women (Table 1). To evaluate physical ability, Cruz-Jentoft et al and Chen et al determined cutoff values for gait speed and/or grip strength, while Fielding et al and Morley et al established limits for gait speed only. Finally, the Studenski et al's definition is based on a sex-related grip strength criterion (Table 1).

\section{Screening for sarcopenia: five methods identified}

Despite the growing interest in sarcopenia study, a scarcity of screening tests for sarcopenia is available. Currently, we identified five available screening methods validated across the scientific literature: the two-step algorithm of the EWGSOP, ${ }^{18}$ the SARC-F questionnaire by Malmstrom et $\mathrm{al},{ }^{23}$ the screening grid from Goodman et al, ${ }^{24}$ the score chart of Ishii et $\mathrm{al}^{25}$ and the prediction equation of Yu et al. ${ }^{26}$ A concise presentation of these tests is presented in Table 2.

Still other screening methods are available to screen for sarcopenia (e.g., measuring calf circumference ${ }^{27}$ or using chair and stand test ${ }^{28}$ ), but they focused only on a particular sex or have not been validated and have therefore been excluded from the current analysis.

\section{Other parameters investigated during the interviews}

In the SarcoPhAge study, each year of follow-up, a very large number of anamnestic, sociodemographic, anthropometric and clinical data as well as physical tests are collected by a well-trained clinical research assistant. In the following paragraph, we describe the main examinations that are useful for the diagnosis of sarcopenia and its screening.

First, body height and weight were gathered using standardized instrument to the nearest $0.1 \mathrm{~cm}$ and $0.1 \mathrm{~kg}$, respectively, and body mass index (BMI) was therefore calculated as the weight divided by the height squared $\left(\mathrm{kg} / \mathrm{m}^{2}\right)$. Calf, wait, wrist and arm circumferences were also recorded at the nearest $0.1 \mathrm{~cm}$. The level of education was documented. The subjects were also asked to list all comorbidities they were suffering from and all current medications taken. Moreover, to assess the cognitive function, we applied the Mini-Mental State Examination (MMSE), scored on up to 30 points. ${ }^{29}$

\section{Statistical methods}

First, descriptive statistics were used to describe our population of older subjects with regard to their main characteristics. The results are expressed as mean $\pm \mathrm{SD}$ for continuous

Table I Five operational definitions of sarcopenia and the cutoff limits applied

\begin{tabular}{|c|c|c|c|}
\hline Diagnosis definition & Muscle mass & Muscle strength & Physical performance \\
\hline Cruz-Jentoft et al ${ }^{18}$ & Women: $\mathrm{SMI} \leq 5.50 \mathrm{~kg} / \mathrm{m}^{2}$ & Women: handgrip strength $<20 \mathrm{~kg}$ & $\mathrm{SPPB} \leq 8$ \\
\hline (EWGSOP) & Men: $\mathrm{SMI} \leq 7.26 \mathrm{~kg} / \mathrm{m}^{2}$ & Men: handgrip strength $<30 \mathrm{~kg}$ & \\
\hline Fielding et al ${ }^{19}$ & Women: SMI $\leq 5.67 \mathrm{~kg} / \mathrm{m}^{2}$ & Not applicable & Gait speed $<1.0 \mathrm{~m} / \mathrm{s}$ \\
\hline (IWGS) & Men: SMI $\leq 7.23 \mathrm{~kg} / \mathrm{m}^{2}$ & & \\
\hline Morley et $\mathrm{a}^{20}$ & Women: SMI $\leq 5.18 \mathrm{~kg} / \mathrm{m}^{2}$ & Not applicable & Gait speed $<1.0 \mathrm{~m} / \mathrm{s}$ \\
\hline (Society of Sarcopenia, Cachexia and & Men: SMI $\leq 6.8 \mathrm{l} \mathrm{kg} / \mathrm{m}^{2}$ & & \\
\hline \multicolumn{4}{|l|}{ Wasting Disorders) } \\
\hline Chen et $\mathrm{al}^{21}$ & Women: $\mathrm{SMI} \leq 5.40 \mathrm{~kg} / \mathrm{m}^{2}$ & Women: handgrip strength $<18 \mathrm{~kg}$ & Gait speed $<0.8 \mathrm{~m} / \mathrm{s}$ \\
\hline (AWGS) & Men: $S M I \leq 7.00 \mathrm{~kg} / \mathrm{m}^{2}$ & Men: handgrip strength $<26 \mathrm{~kg}$ & \\
\hline Studenski et $\mathrm{a}^{22}$ & Women: $\mathrm{ALM}_{\mathrm{BM}}<0.5 \mathrm{I} 2$ & Women: handgrip strength $<16 \mathrm{~kg}$ & Not applicable \\
\hline (FNIH Sarcopenia Project) & Men: $\mathrm{ALM}_{\mathrm{BMI}}<0.789$ & Men: handgrip strength $<26 \mathrm{~kg}$ & \\
\hline
\end{tabular}

Abbreviations: ALM, appendicular skeletal lean mass; AWGS, Asian Working Group for Sarcopenia; BMI, body mass index; EWGSOP, European Working Group on Sarcopenia in Older People; IWGS, International Working Group on Sarcopenia; SMI, skeletal muscle mass index; SPPB, Short Physical Performance Battery. 
Table 2 Description of five screening strategies for sarcopenia

\begin{tabular}{|c|c|c|c|c|}
\hline Study team & $\begin{array}{l}\text { Type of } \\
\text { screening tools }\end{array}$ & Aim of the tool & Principle and variables of interest & $\begin{array}{l}\text { Authors' categorization in } \\
\text { "at risk" or "not at risk" of } \\
\text { sarcopenia }\end{array}$ \\
\hline $\begin{array}{l}\text { Cruz-Jentoft } \\
\text { et al }\left.\right|^{18}\end{array}$ & $\begin{array}{l}\text { Two-stage } \\
\text { algorithm }\end{array}$ & $\begin{array}{l}\text { To enable rapid } \\
\text { identification of individuals } \\
\text { who should undergo a } \\
\text { thorough examination (i.e., } \\
\text { measurement of muscle } \\
\text { mass using DEXA) for the } \\
\text { diagnosis of sarcopenia }\end{array}$ & $\begin{array}{l}\text { Assessment of gait speed: if gait speed } \\
\text { is too slow }(\leq 0.8 \mathrm{~m} / \mathrm{s}) \text {, measure muscle } \\
\text { mass. } \\
\text { If gait speed is }>0.8 \mathrm{~m} / \mathrm{s} \text {, proceed to } \\
\text { an assessment of grip strength: if grip } \\
\text { strength is low ( }<20 \mathrm{~kg} \text { for female, }<30 \mathrm{~kg} \\
\text { for men), measure muscle mass }\end{array}$ & $\begin{array}{l}\text { Subjects presenting a low gait } \\
\text { speed or a low gait speed plus a } \\
\text { low grip strength are considered } \\
\text { "at risk of sarcopenia" }\end{array}$ \\
\hline $\begin{array}{l}\text { Malmstrom } \\
\text { et } \mathrm{al}^{23}\end{array}$ & $\begin{array}{l}\text { SARC-F } \\
\text { questionnaire }\end{array}$ & $\begin{array}{l}\text { To rapidly identify } \\
\text { individuals who require a } \\
\text { diagnostic examination for } \\
\text { sarcopenia }\end{array}$ & $\begin{array}{l}\text { Five-domain symptom-based } \\
\text { questionnaire: strength, ambulation } \\
\text { (walking independence), rising from a } \\
\text { chair, stair climbing and history of falls. } \\
\text { The total score is } 10 \text { points (with each } \\
\text { component scoring } 2 \text { ) }\end{array}$ & $\begin{array}{l}\text { A score of } \geq 4 \text { points is predictive } \\
\text { of sarcopenia }\end{array}$ \\
\hline $\begin{array}{l}\text { Goodman } \\
\text { et } \mathrm{al}^{24}\end{array}$ & Screening grid & $\begin{array}{l}\text { To use predictors of low } \\
\text { muscle mass to identify } \\
\text { subjects requiring a } \\
\text { diagnostic evaluation of low } \\
\text { muscle mass (using DEXA) }\end{array}$ & $\begin{array}{l}\text { Screening grid built using age and BMI and } \\
\text { developed for both men and women, It } \\
\text { provides, according to the age and the BMI } \\
\text { of the subject, the probability (\%) of low } \\
\text { muscle mass }\end{array}$ & $\begin{array}{l}\text { Individuals with a probability (given } \\
\text { by the grid) above } 70 \% \text { in men } \\
\text { and above } 80 \% \text { in women are } \\
\text { considered as having low muscle } \\
\text { mass (i.e., at risk of sarcopenia) }\end{array}$ \\
\hline $\mid s h i i$ et a| $\left.\right|^{25}$ & Score chart & $\begin{array}{l}\text { To identify older adults at } \\
\text { high risk of sarcopenia }\end{array}$ & 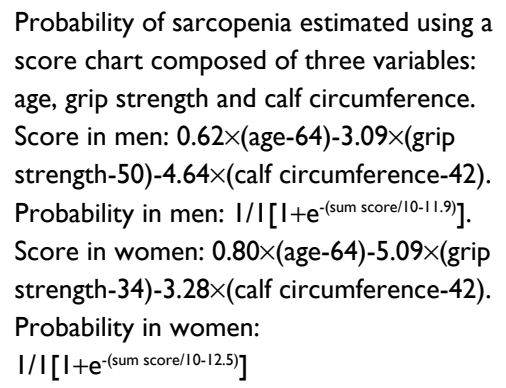 & $\begin{array}{l}\text { Sum score above } 105 \text { in men } \\
\text { and } 120 \text { in women determines } \\
\text { people having a high probability of } \\
\text { sarcopenia }\end{array}$ \\
\hline Yu et $\mathrm{al}^{26}$ & $\begin{array}{l}\text { Anthropometric } \\
\text { prediction } \\
\text { equation }\end{array}$ & $\begin{array}{l}\text { To determine a prediction } \\
\text { equation of a low muscle } \\
\text { mass }\end{array}$ & $\begin{array}{l}\text { Anthropometric prediction equation based } \\
\text { on four parameters: weight, BMI, age and } \\
\text { sex. } \\
\text { Appendicular skeletal muscle mass } \\
\text { prediction equation: } 10.05+0.35 \text { (weight)- } \\
0.62 \text { (BMI)- } 0.02 \text { (age) }+5.10 \text { (if male) }\end{array}$ & $\begin{array}{l}\text { Subjects presenting a score, } \\
\text { derived from the prediction } \\
\text { equation, below the 20th } \\
\text { percentile value (computed for our } \\
\text { cohort) were considered "at risk } \\
\text { of sarcopenia" }\end{array}$ \\
\hline
\end{tabular}

Abbreviations: BMI, body mass index; DEXA, dual-energy X-ray absorptiometry; EWGSOP, European Working Group on Sarcopenia in Older People.

variables, normality being checked using Shapiro-Wilk test. Absolute and relative (\%) frequencies were reported for qualitative variables.

Furthermore, to measure the agreement between the five diagnostic definitions of sarcopenia, we reported the Cohen's kappa coefficients (concordance rate, two by two) and the Fleiss' kappa coefficient (overall concordance rate). A coefficient less than 0 indicates disagreement. A coefficient between 0 and 0.20 reflects a slight agreement. The agreement is fair for a value between 0.21 and 0.40 , moderate between 0.41 and 0.60 and substantial between 0.61 and 0.80 . An almost perfect agreement exists when the value is between 0.81 and $1 .^{30}$

Subsequently, the clinical validity of the five screening approaches was measured using four indicators of performance: sensitivity, specificity, positive predictive value (PPV) and negative predictive value (NPV). ${ }^{31}$ For the diagnosis of sarcopenia, these are the five operational definitions that were considered the standard criteria. To determine whether the screening test is positive or negative (i.e., to obtain a binary outcome from the quantitative result to the screening tool), we used the specific cutoffs proposed by the authors of these screening tools in their study work ${ }^{18,23-26}$ (Table 2). The sensitivity represents the proportion of subjects actually presenting with sarcopenia (based on the reference diagnosis), having been correctly identified as sarcopenic using the screening tool (i.e., positive screening test). The specificity is the proportion of subjects who do not actually have sarcopenia (based on the clinical diagnosis), which were correctly identified as non-sarcopenic using the screening tool 
(i.e., negative screening test). The PPV is the probability of presenting sarcopenia in case of a positive screening test, and the NPV is the probability of not suffering from sarcopenia in case of a negative screening test. All of these proportions were presented with their exact $95 \%$ CI.

Thereafter, we performed an extensive analysis of the tool performance by measuring the association between the five screening methods and the five diagnosis definitions of sarcopenia. A stepwise binary logistic regression was carried out to study the association between each definition of sarcopenia and each of the five screening tools. An odds ratio (OR) with $95 \% \mathrm{CI}$ was calculated. An OR $>1$ indicates that the parameter is a risk factor for sarcopenia, while an $\mathrm{OR}<1$ indicates that the parameter is a protective factor. Adjustments for age, sex, number of comorbidities, number of drugs and cognitive status were performed. These covariates were included in the multivariable model because they significantly differed between groups in the univariate analysis for at least one of the diagnostic classifications of sarcopenia. No adjustment was performed for BMI to avoid overadjustment because of being directly related to the amount of muscle mass.

For four out of the five screening tools analyzed (Malmstrom et al, Goodman et al, Ishii et al and Yu et al), a receiver operating characteristic (ROC) analysis allowed us to determine the area under the curve (AUC). Indeed, this type of analysis could not be performed for the two-stage algorithm of the EWGSOP because this tool only furnishes dichotomous results to the screening test. Thenceforth, quantitative results of the four screening tools and dichotomized results of the diagnosis of sarcopenia were used to compute the AUC value (and its $95 \% \mathrm{CI}$ ). An AUC value under 0.5 reflects no discriminatory power, while an AUC between 0.5 and 1.0 is thus essential for clinical testing. ${ }^{32}$ An AUC closer to 1 demonstrates a higher screening power; the tool presenting the highest AUC value was then considered to perform better at distinguishing very well those at risk of sarcopenia compared to those not at risk. The AUC values were statistically compared among the four screening tools using the method developed by Delong et al: ${ }^{33}$ a $p$-value $<0.05$ indicates that the AUC values significantly differ among them. The AUC was represented using the ROC curves. In addition, for each screening tool and each definition of the sarcopenia, a new optimal cutoff has been calculated according to different statistical methods: the point that minimizes the distance between the ROC curve and the perfect point (distance 0.1 ) and the Youden's index (i.e., maximum [sensitivity + specificity - 1]). The cutoff values derived from Youden's index seem recognized as more sensitive. ${ }^{34}$
The overall results were considered statistically significant at the $5 \%$ critical level. The statistical analyses were performed using SPSS Statistics 24 (IBM Corporation, Armonk, NY, USA) and SAS 9.4 (SAS Institute Inc., Cary, $\mathrm{NC}$, USA) software packages.

\section{Results}

\section{Description and characterization of the study sample}

As shown in Figure 1, out of the 534 subjects initially enrolled in the SarcoPhAge study, ${ }^{5} 336$ were reviewed during the 2-year follow-up, and 306 older adults had complete necessary data and were included in this ancillary analysis. Individuals were $74.8 \pm 5.9$ years of age, and the cohort comprised 182 women (59.5\%). A summary of baseline characteristics is given in Table 3. The demographic and clinical characteristics of the subjects analyzed in the current analysis do not differ significantly from those of the participants excluded due to missing data (all $p$-values $>0.05$ ).

Among the 306 older adults assessed, between 17 and 51 subjects were diagnosed as sarcopenic depending on the diagnostic definition applied. The prevalence of sarcopenia thus varied from $5.6 \%$ (Chen et al) to $16.7 \%$ (Cruz-Jentoft et al). The measures of agreement between the diagnostic definitions are provided in Table 4. Results revealed a slight to moderate agreement across diagnostic definitions, except the substantial agreement observed when comparing the concordance between the definition of Cruz-Jentoft et al and the diagnostic criterion of Fielding et al (Cohen's kappa coefficient was $0.71,95 \%$ CI $0.66-0.76$ ). Furthermore, a moderate overall Fleiss' kappa coefficient was observed (0.42, 95\% CI 0.39-0.46), which means that, globally, the five diagnostic definitions are moderately concordant. We also tested the agreement rate between the five screening methods (Table 4). A poor-to-moderate agreement was observed when comparing each tool two by two. The overall agreement reached 0.27 (i.e., a globally fair concordance rate between screening methods).

\section{Performance of the screening tests}

We first established, for each screening instrument, its sensitivity, specificity, PPV and NPV. As summarized in Table 5, the best sensitivity (up to 100\%) and the best NPV (up to $100 \%$ ) was obtained with the screening test of Ishii et al, regardless of the definition tested. The most specific tool was the two-stage algorithm of the EWGSOP (88.5-91.1\% depending on the operational definition tested). Depending on the different diagnostic definitions and the screening tools 


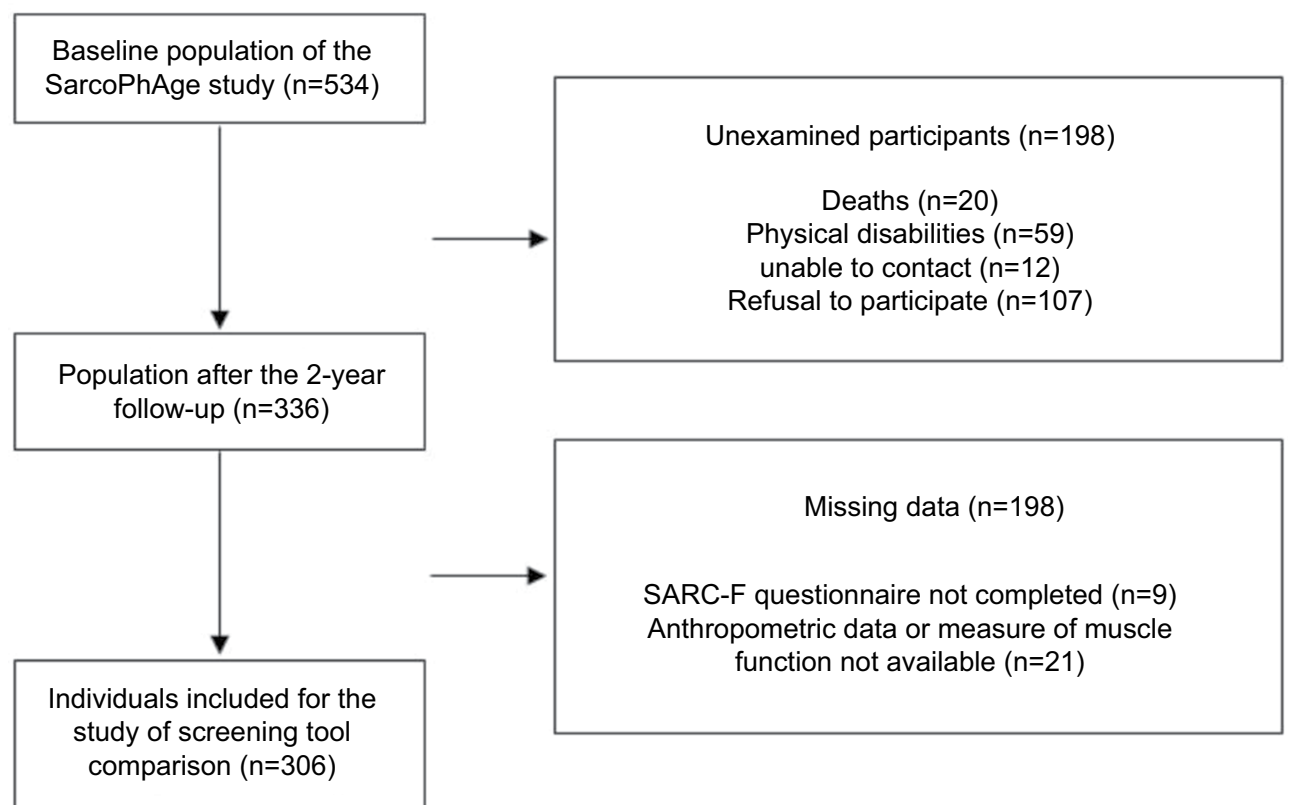

Figure I Description of the participant population analyzed.

Abbreviation: SarcoPhAge, Sarcopenia and Physical Impairment with Advancing Age.

tested, we noted that the magnitude of the sensitivity value of the tool could vary widely: from $5.88 \%$ for the screening grid of Goodman et al (against Studenski et al's diagnostic criteria) to $100 \%$ for the tool of Ishii et al (against Morley et al's diagnostic criteria). More particularly, for the same screening tool, the sensitivity values were sometimes very remote according to the sarcopenia definition applied: for example, the sensitivity of the two-step algorithm of the EWGSOP passing from $33.3 \%$ for the definition of CruzJentoft et al to $70.6 \%$ for the definition of Chen et al. With regard to specificity, it was always above $60 \%$ across all the diagnostic definitions envisaged, thus demonstrating a good capacity of all the tools to identify elders without sarcopenia. For the set of screening tools, all NPVs were superior to $87.0 \%$, regardless of the diagnosis criteria concerned, indicating a good probability of not suffering from sarcopenia in the case of a negative screening test. However, in each case, all PPVs (which represent the probability of presenting sarcopenia in the case of a positive screening test) were below $51.0 \%$, with a minimum of $4.26 \%$ (when applying the definition of Studenski et al) and a maximum of $50.9 \%$ (when applying the definition of Cruz-Jentoft et al) for the same screening tool (that of Goodman et al). The same tool could thus demonstrate the lowest and the highest PPV, depending on the diagnostic definition.

Subsequently, through an extensive analysis (Table 6), we observed that every screening strategy was significantly associated with the different definitions of sarcopenia: all $p$-values were at least inferior to 0.05 , after adjustments for potential confounders. For most of the tools, this association was positive: the higher the score obtained with the screening test, the greater the risk of sarcopenia. It was normal to consistently note a negative association for the equation of $\mathrm{Yu}$ et al, because, in this case, the lower the score, the greater the risk of being sarcopenic. However, a negative association was observed for the screening grid of Goodman et al only when applying the definition of Studenski et al, but in this case, the OR was really close to 1 , which meant no association. It is the binary algorithm tool of the EWGSOP that showed the highest ORs (up to 19.8 according to the definition). Among the four other tools using quantitative results, the SARC-F questionnaire appears to show significantly high ORs (except for the definition of Morley et al): the higher the score for the SARC-F tool, the greater the risk of suffering from sarcopenia.

Thereafter, for all screening approaches for which we could perform ROC analysis (four out of the five identified), we noticed that all AUC values were superior to the point with no discriminant power $(0.500)$ regardless of the definition employed, with a minimum equal to 0.600 for the screening grid of Goodman et al. The highest AUC has been demonstrated with the tool of Ishii et al: $0.841(0.788-0.894)$ for the diagnostic definition of Fielding et al to 0.914 (0.873-0.956) for the diagnostic definition of Chen et al. The AUC values of the chart score of Ishii et al significantly differed, in a majority of the cases, from the AUC values of the other tools, 
Table 3 Summary of participant characteristics $(n=306)$

\begin{tabular}{|c|c|c|}
\hline Characteristics & Men $(n=124)$ & Women $(n=182)$ \\
\hline Age (years) & $75.0 \pm 5.9$ & $74.7 \pm 5.9$ \\
\hline \multicolumn{3}{|l|}{ Anthropometric data } \\
\hline Weight (kg) & $|7| .6 \pm 6.7$ & $157.9 \pm 6.8$ \\
\hline Height (cm) & $82.0 \pm 14.3$ & $65.7 \pm 13.5$ \\
\hline BMI $\left(\mathrm{kg} / \mathrm{m}^{2}\right)$ & $27.8 \pm 4.3$ & $26.3 \pm 4.9$ \\
\hline Calf circumference $(\mathrm{cm})$ & $35.8 \pm 3.2$ & $33.7 \pm 3.4$ \\
\hline Waist circumference $(\mathrm{cm})$ & $98.6 \pm 12.6$ & $87.0 \pm 12.7$ \\
\hline Wrist circumference $(\mathrm{cm})$ & $17.9 \pm 1.2$ & $|5.8 \pm 1|$. \\
\hline Arm circumference (cm) & $28.5 \pm 3.1$ & $27.2 \pm 3.2$ \\
\hline \multicolumn{3}{|l|}{ Level of education } \\
\hline Without qualification & $3(2.4)$ & $2(1.1)$ \\
\hline Primary school & $10(8.1)$ & $18(9.9)$ \\
\hline Secondary school & $53(42.8)$ & $94(5 \mathrm{I} .7)$ \\
\hline Postsecondary education & $55(44.3)$ & $65(35.7)$ \\
\hline Doctorate & $3(2.4)$ & $3(1.6)$ \\
\hline $\begin{array}{l}\text { Number of concomitant } \\
\text { diseases by subject }\end{array}$ & $4.1 \pm 2.6$ & $4.3 \pm 2.4$ \\
\hline $\begin{array}{l}\text { Number of drugs consumed by } \\
\text { subject }\end{array}$ & $5.9 \pm 3.2$ & $6.8 \pm 3.8$ \\
\hline MMSE (/30 points) & $28.7 \pm 1.5$ & $28.8 \pm 2.0$ \\
\hline SMI $\left(\mathrm{kg} / \mathrm{m}^{2}\right)$ & $7.9 \pm 1.0$ & $6.1 \pm 0.9$ \\
\hline Muscle strength (kg) & $37.6 \pm 8.7$ & $19.6 \pm 6.2$ \\
\hline SPPB (/I2 points) & $10.2 \pm 1.1$ & $9.6 \pm 1.2$ \\
\hline $4 \mathrm{~m}$ gait speed $(\mathrm{m} / \mathrm{s})$ & $1.2 \pm 0.3$ & $1.0 \pm 0.3$ \\
\hline Prevalence of sarcopenia using & $19(15.3)$ & $32(17.6)$ \\
\hline \multicolumn{3}{|l|}{ Cruz-Jentoft et al's criteria } \\
\hline $\begin{array}{l}\text { Prevalence of sarcopenia using } \\
\text { Fieldling et al's criteria }\end{array}$ & $16(12.9)$ & $21(11.5)$ \\
\hline Prevalence of sarcopenia using & $8(6.4)$ & $10(5.5)$ \\
\hline \multicolumn{3}{|l|}{ Morley et al's criteria } \\
\hline Prevalence of sarcopenia using & $5(4.0)$ & $12(6.6)$ \\
\hline \multicolumn{3}{|l|}{ Chen et al's criteria } \\
\hline Prevalence of sarcopenia using & $7(5.6)$ & $15(8.2)$ \\
\hline Studenski et al's criteria & & \\
\hline
\end{tabular}

Abbreviations: BMI, body mass index; MMSE, Mini-Mental State Examination; SMI, skeletal muscle mass index; SPPB, Short Physical Performance Battery. showing that the performance of the screening tool of Ishii et al was significantly better than that demonstrated by the other tools. For each definition, the ROC curves, as shown in Figure 2, represented visually the differences in the screening tool performances.

\section{Optimal cutoff limits for screening tools}

Finally, for the four tests for which cutoff points could be calculated, new optimal cutoff points were computed (Table 6). In most situations, the thresholds provided are similar or even identical depending on the use of a particular statistical method (i.e., distance 0.1 or Youden's index), except in the case of the screening grid of Goodman et al using the Cruz-Jentoft et al's diagnostic criteria. It was also for this tool only that we observed a great variation, across the different diagnosis definitions, in the proposed cutoffs: it changed from a proposed cutoff of $16.8 \%$ when applying the definition of Cruz-Jentoft et al to a proposed cutoff of $73.3 \%$ when the Chen et al's definition was applied. Note that the initial cutoff proposed by Goodman et al to determine a low muscle mass had a probability of $70 \%$ (for men) or $80 \%$ (for women; Table 2). Concerning the SARC-F questionnaire of Malmstrom et al, we observed the same cutoff proposed when using the diagnostic definition of Morley et al and that of Studenski et al: a score of 4 or more is predictive of sarcopenia. However, for all the other diagnostic definitions, this cutoff could be lowered by two points out of 10. For the chart of Ishii et al, a cutoff value higher than that proposed at baseline could be applied to identify people at risk of sarcopenia: we proposed a cutoff varying from 111.1 to 128.5 for the sum score compared to 105 for men and 120 for women proposed initially

Table 4 Concordance between the five diagnostic definitions of sarcopenia and between the five screening methods

\begin{tabular}{|c|c|c|c|c|c|}
\hline Diagnostic definitions & $\begin{array}{l}\text { Definition of } \\
\text { Cruz-Jentoft et al }\end{array}$ & $\begin{array}{l}\text { Definition of } \\
\text { Fielding et al }\end{array}$ & $\begin{array}{l}\text { Definition of } \\
\text { Morley et al }\end{array}$ & $\begin{array}{l}\text { Definition of } \\
\text { Chen et al }\end{array}$ & $\begin{array}{l}\text { Definition of } \\
\text { Studenski et al }\end{array}$ \\
\hline Definition of Cruz-Jentoft et al & I & $0.71(0.66-0.76)$ & $0.44(0.38-0.50)$ & $0.45(0.39-0.5 \mathrm{I})$ & $0.22(0.17-0.27)$ \\
\hline Definition of Fielding et al & & 1 & $0.62(0.57-0.67)$ & $0.56(0.50-0.62)$ & $0.20(0.15-0.24)$ \\
\hline Definition of Morley et al & & & 1 & $0.48(0.42-0.57)$ & $0.14(0.10-0.18)$ \\
\hline Definition of Chen et al & & & & 1 & $0.26(0.2 I-0.3 I)$ \\
\hline Definition of Studenski et al & & & & & 1 \\
\hline Screening tools & $\begin{array}{l}\text { Two-stage algorithm } \\
\text { of the EWGSOP }\end{array}$ & $\begin{array}{l}\text { SARC-F of } \\
\text { Malmstrom et al }\end{array}$ & $\begin{array}{l}\text { Screening grid of } \\
\text { Goodman et al }\end{array}$ & $\begin{array}{l}\text { Score chart of } \\
\text { Ishii et al }\end{array}$ & $\begin{array}{l}\text { Equation of } \\
\text { Yu et al }\end{array}$ \\
\hline $\begin{array}{l}\text { Two-stage algorithm of the } \\
\text { EWGSOP }\end{array}$ & 1 & $0.46(0.40-0.52)$ & $0.05(0.03-0.07)$ & $0.43(0.37-0.48)$ & $0.17(0.13-0.21)$ \\
\hline SARC-F of Malmstrom et al & & 1 & $0.05(0.03-0.07)$ & $0.29(0.24-0.34)$ & $0.13(0.09-0.17)$ \\
\hline Screening grid of Goodman et al & & & 1 & $0.18(0.14-0.22)$ & $0.63(0.58-0.68)$ \\
\hline Score chart of Ishii et al & & & & 1 & $0.31(0.26-0.36)$ \\
\hline Equation of $\mathrm{Yu}$ et al & & & & & 1 \\
\hline
\end{tabular}

Abbreviation: EWGSOP, European Working Group on Sarcopenia in Older People. 
Table 5 Indicators of performance of five screening methods across five definitions of sarcopenia $(n=306)$

\begin{tabular}{|c|c|c|c|c|}
\hline $\begin{array}{l}\text { Screening method according to } \\
\text { diagnosis definition }\end{array}$ & $\begin{array}{l}\text { Sensitivity, proportion, } \\
\%(95 \% \mathrm{Cl})\end{array}$ & $\begin{array}{l}\text { Specificity, proportion, } \\
\%(95 \% \mathrm{Cl})\end{array}$ & $\begin{array}{l}\text { PPV, probabilit, } \\
\%(95 \% \mathrm{Cl})\end{array}$ & $\begin{array}{l}\text { NPV, probability, } \\
\%(95 \% \mathrm{CI})\end{array}$ \\
\hline \multicolumn{5}{|l|}{ Definition of Cruz-Jentoft et al } \\
\hline Two-stage algorithm of the EWGSOP & $33.3(28.0-38.6)$ & $91.0(87.8-94.2)$ & $42.5(37.0-48.0)$ & $87.2(83.5-90.9)$ \\
\hline SARC-F of Malmstrom et al & $36.0(30.6-41.4)$ & $87.1(83.3-90.9)$ & $35.3(29.9-40.7)$ & $87.4(83.7-91.1)$ \\
\hline Screening grid of Goodman et al & $47.5(41.9-53.1)$ & $89.4(86.0-92.8)$ & $50.9(45.3-56.5)$ & $88.0(84.4-91.6)$ \\
\hline Score chart of Ishii et al & $84.3(80.2-88.4)$ & $80.9(76.5-85.3)$ & $46.7(4||-52.3)$. & $96.3(94.2-98.4)$ \\
\hline Equation of Yu et al & $51.0(45.4-56.6)$ & $86.7(82.9-90.5)$ & $43.3(37.7-48.9)$ & $89.8(86.4-93.2)$ \\
\hline \multicolumn{5}{|l|}{ Definition of Fielding et al } \\
\hline Two-stage algorithm of the EWGSOP & $43.2(37.6-48.8)$ & 9I.I (87.9-94.3) & $40.0(34.5-45.5)$ & $92.1(89.1-95.1)$ \\
\hline SARC-F of Malmstrom et al & $43.2(37.6-48.8)$ & $86.6(82.8-90.4)$ & $30.8(25.6-36.0)$ & $91.7(88.6-94.8)$ \\
\hline Screening grid of Goodman et al & $45.9(40.3-51.5)$ & $88.8(85.3-92.3)$ & $36.2(30.8-41.6)$ & $92.3(89.3-95.3)$ \\
\hline Score chart of Ishii et al & $86.8(83.0-90.6)$ & $77.7(73.0-82.4)$ & $34.8(29.5-40.1)$ & $97.7(96.0-99.4)$ \\
\hline Equation of $\mathrm{Yu}$ et al & $64.9(59.6-70.2)$ & $86.6(82.8-90.4)$ & $40.0(34.5-45.5)$ & $94.7(92.2-97.2)$ \\
\hline \multicolumn{5}{|l|}{ Definition of Morley et al } \\
\hline Two-stage algorithm of the EWGSOP & $38.9(33.4-44.4)$ & $88.5(84.9-92.1)$ & $17.5(|3.2-2| .8)$ & $95.9(93.7-98.1)$ \\
\hline SARC-F of Malmstrom et al & $55.6(50.0-6 \mid .2)$ & $85.4(81.4-91.4)$ & $19.2(\mid 4.8-23.6)$ & $96.8(94.8-98.8)$ \\
\hline Screening grid of Goodman et al & $66.7(61.4-72.0)$ & $87.8(84.1-91.5)$ & $25.5(20.6-30.4)$ & $97.7(96.0-99.4)$ \\
\hline Score chart of Ishii et al & $100.0(100-100)$ & $74.3(69.4-79.2)$ & $34.8(29.5-40.1)$ & $97.7(96.0-99.4)$ \\
\hline Equation of Yu et al & $83.3(79.1-87.5)$ & $84.4(80.3-88.5)$ & $25.0(20.1-29.9)$ & $98.8(97.6-100)$ \\
\hline \multicolumn{5}{|l|}{ Definition of Chen et al } \\
\hline Two-stage algorithm of the EWGSOP & $70.6(65.5-75.7)$ & $90.3(87.0-93.6)$ & $30.0(24.9-35.1)$ & 98.1 (96.6-99.6) \\
\hline SARC-F of Malmstrom et al & $52.9(47.3-58.5)$ & $85.1(81.1-89.1)$ & $17.3(|3.1-2| .5)$ & $96.8(94.8-98.8)$ \\
\hline Screening grid of Goodman et al & $41.2(35.7-46.7)$ & $86.2(82.3-90.1)$ & $14.9(10.9-18.9)$ & 96.1 (93.9-98.3) \\
\hline Score chart of Ishii et al & $100.0(100-100)$ & $74.1(69.2-79.0)$ & $14.5(10.6-18.4)$ & $100.0(100-100)$ \\
\hline Equation of $\mathrm{Yu}$ et al & I6.1 (I2.0-20.2) & $60.0(54.5-65.5)$ & $42.0(38.9-45.1)$ & $91.1(87.9-94.3)$ \\
\hline \multicolumn{5}{|l|}{ Definition of Studenski et al } \\
\hline Two-stage algorithm of the EWGSOP & $50.0(44.4-55.6)$ & $89.8(86.4-93.2)$ & $27.5(22.5-32.5)$ & $95.9(93.6-98.0)$ \\
\hline SARC-F of Malmstrom et al & $40.9(35.4-46.4)$ & $84.9(80.9-88.9)$ & $17.3(|3|-2 \mid .5)$. & $94.9(92.4-97.4)$ \\
\hline Screening grid of Goodman et al & $5.88(3.20-8.50)$ & $83.5(79.3-87.7)$ & $4.26(2.0-6.50)$ & $87.6(83.9-91.3)$ \\
\hline Score chart of Ishii et al & $90.9(87.7-94.1)$ & $74.9(70.0-79.8)$ & $21.7(17.1-26.3)$ & $99.1(98.0-100)$ \\
\hline Equation of Yu et al & $36.4(3|.0-4| .8)$ & $81.7(77.4-86.0)$ & I3.3 (9.50-17.I) & $94.3(91.7-96.9)$ \\
\hline
\end{tabular}

Abbreviations: EWGSOP, European Working Group on Sarcopenia in Older People; NPV, negative predictive value; PPV, positive predictive value.

(Table 2). Finally, for the screening tool of Yu et al, the new cutoffs seemed to be substantially similar to those originally developed (Table 2): a value inferior to approximately 15 to the result of the predictive equation seems to predict a low muscle mass.

\section{Discussion}

The aim of the current study was to evaluate the performance of five screening tools for sarcopenia by comparing their psychometric properties conforming to different diagnostic definitions of the condition using data obtained in the SarcoPhAge cohort.

A criterion of an ideal screening test is to demonstrate reasonably accurate sensitivity and specificity. ${ }^{31}$ In our analyses, all the tools globally showed a poor sensitivity but an excellent specificity, in agreement with the performance established in the initial validations. ${ }^{23-26}$ Moreover, the NPVs were consistently high. We can then conclude that all the screening tools performed well in identifying the subjects who do not suffer from sarcopenia and who should not, with certainty, benefit from further assessment of muscle mass, avoiding unnecessary or disadvantageous diagnostic investigations. However, the screening tools evaluated, because suffering from low levels of sensitivity rate, do not have the necessary performance to prove to be efficient screening instruments in the general population, even if they could be great tests to apply in a population with a high prevalence of sarcopenia (still to be determined). In clinical settings, a high sensitivity rate is important to rapidly identify patients at risk of sarcopenia to propose at the earliest preventive strategies. Therefore, the screening instruments currently available are not entirely efficient in the sense of providing a good ability to identify both cases and non-cases of sarcopenia.

Another approach to establishing a quality parameter of a screening method is the calculation of the AUC value. The set of tools tested in this study demonstrated a good discrimination capacity. Indeed, an AUC value of 0.6, minimum value observed for all tools, is considered sufficient. 


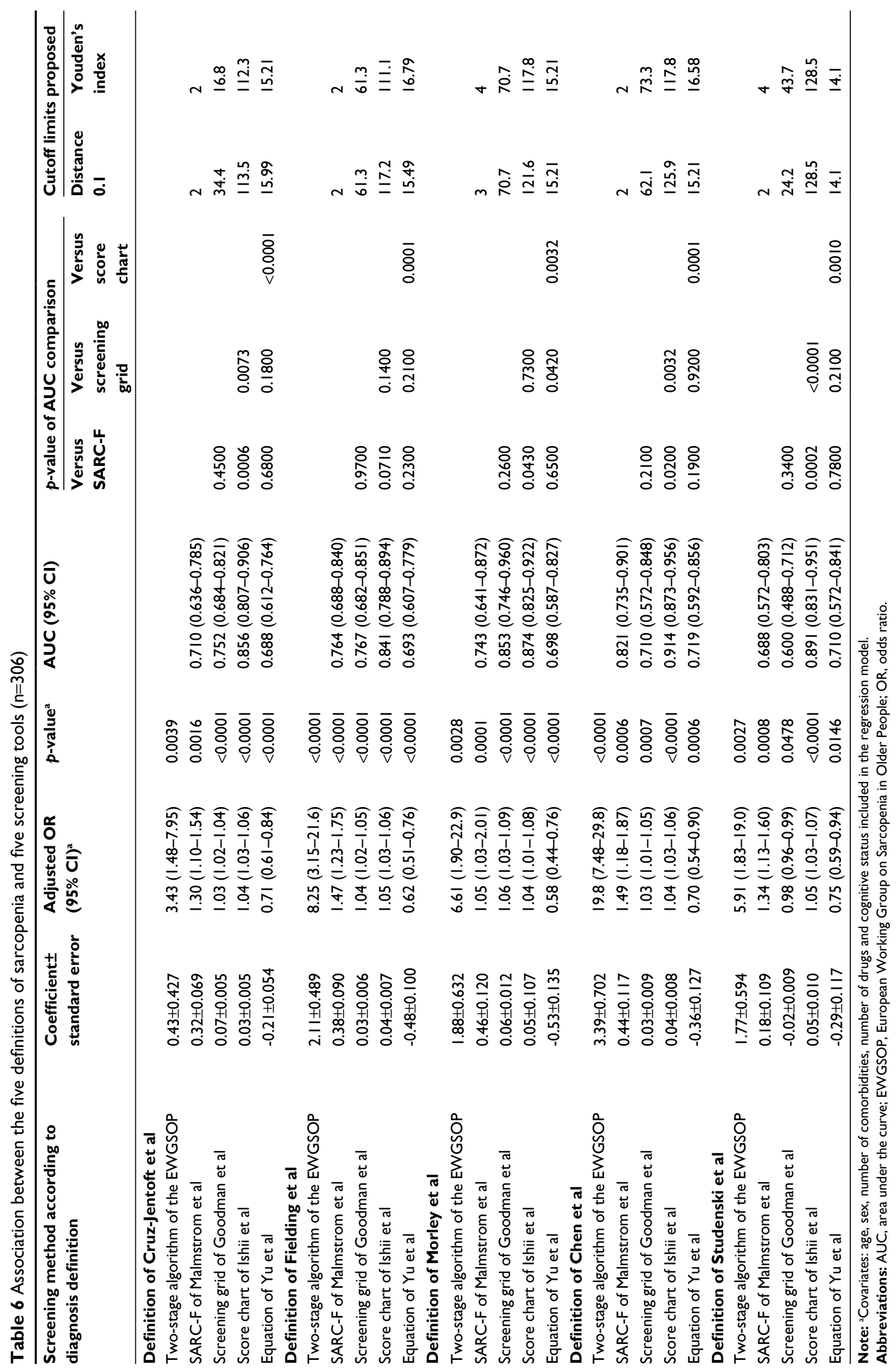


Definition of Cruz-Jentoft et al

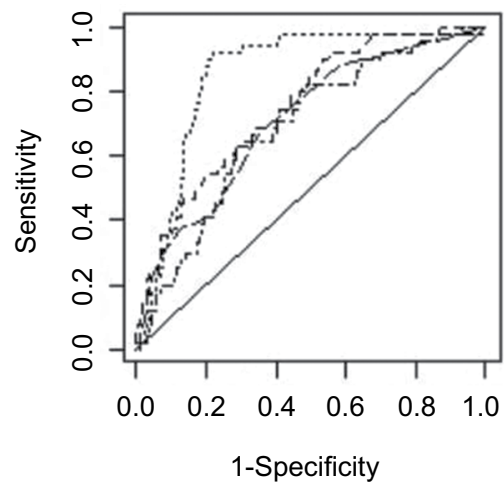

Definition of Chen et al

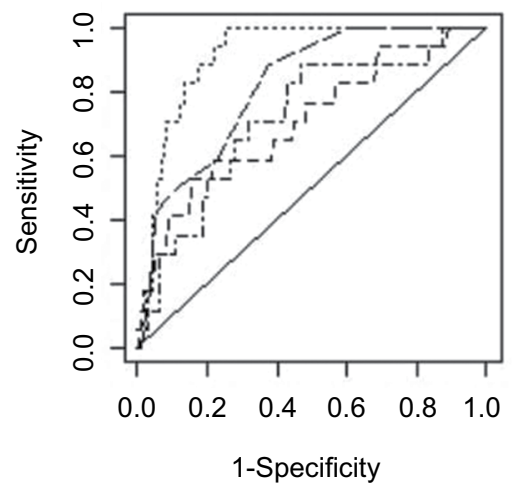

Definition of Fielding et al

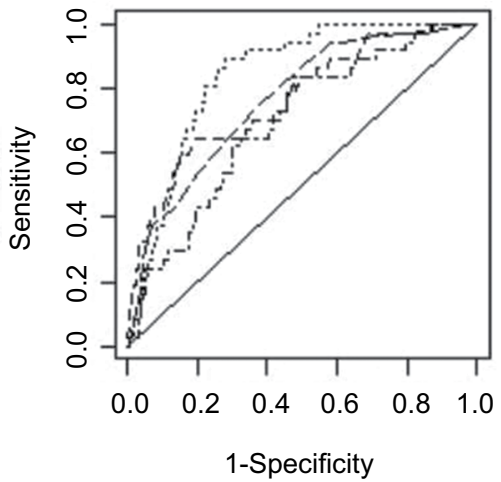

Definition of Studenski et al

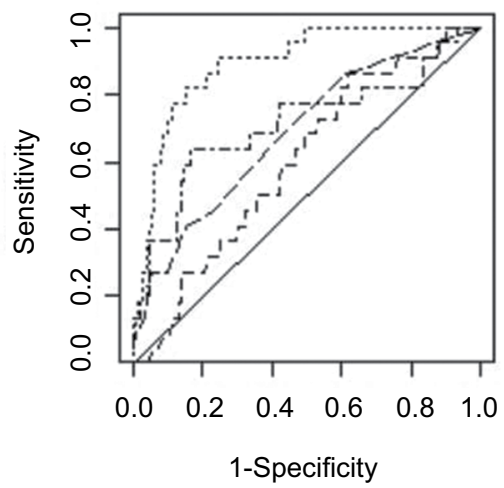

Definition of Morley et al

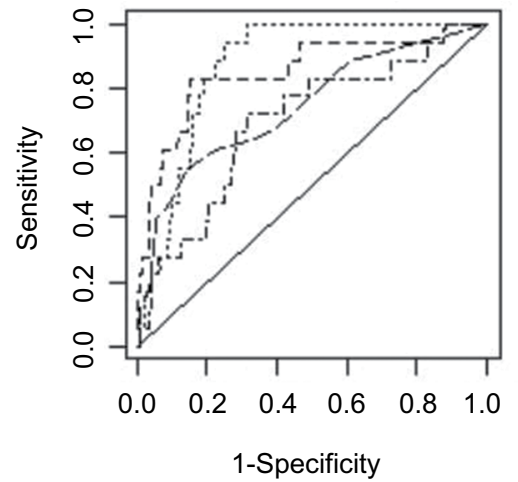

-- SARC-F of Malmstrom et al

-- - Screening grid of Goodman et al

.... Score chart of Ishii et al

...- Equation of Yu et al

Figure 2 ROC curves for comparisons of the five diagnostic definitions of sarcopenia. Abbreviation: ROC, receiver operating characteristic.

More specifically, we highlighted an excellent performance of the tool developed by Ishii et al (AUC value up to 0.9).

In general, the tool of Ishii et al showed thus mathematically better properties. However, it is important to keep in mind that the choice of using a particular screening tool is made according to the means and objectives of the practitioner. For example, the complex and time-consuming calculations required in the tools of Ishii et al and $\mathrm{Yu}$ et al may prove to hinder their use. Indeed, other important efficiency criteria are also to be taken into account (e.g., rapidity of application and simplicity of use and administration). In any case, an extensive analysis allowed to determine that each screening tool is significantly associated with sarcopenia regardless of the diagnostic definition. This reflects the relevance of the use of these tools in clinical practice.

Not surprisingly, we also sometimes noted that the performance and optimal cutoffs proposed vary greatly depending on which definition of sarcopenia was applied. Moreover, the measure of agreement between screening strategies as well as between diagnostic definitions shows a moderate concordance rate meaning that it was not always the same individuals who were screened or diagnosed as sarcopenic.
These two observations reflect the lack of a consensually defined concept of sarcopenia and of standardized screening strategies. It is therefore essential to establish a consensus, which will facilitate standardizations and comparisons in this study field. Indeed, the variations in the characterization of sarcopenia can influence public health policies. For example, an underestimation or overestimation of the prevalence of sarcopenia could affect the therapeutic or preventive interventions likely to be proposed, by increasing the risk of providing unnecessary treatment to a false-positive patient or depriving a false-negative patient from efficient therapeutics. ${ }^{35}$

Through this analysis, we hope to provide a clearer picture of the existing tools that can help the practitioner to investigate the presence or absence of sarcopenia, with the ultimate aim of making every effort to prevent muscular disability, by acting on modifiable factors and lifestyle habits. ${ }^{36}$ This comparative head-to-head study of the screening tools for sarcopenia, in the same population set, had never been performed before.

Moreover, we make in this study possible the easy use of concrete cutoff limits for the various screening tests, choosing across five diagnostic classifications of sarcopenia applicable 
to different populations. However, these cutoffs are given as an indication, recognizing that the added value of providing these new thresholds is limited because our study is itself limited in its external validity.

Indeed, it is necessary to make recommendations concerning the interpretation of our results. Although we used statistical inference techniques, biases may have been introduced, mainly owing to the participant selection process: our sample may not be fully representative. Indeed, our study population was mainly composed of voluntary subjects. These subjects could feel a priori healthier to undertake a 1-hour interview than a random sample of the population. Results may therefore be not fully generalizable. In the same vein, caution should be taken in analyzing the results in light of the comparisons made between screening tests. Indeed, the populations examined are different between our study and the original studies used to develop these tools: researchers proposed cutoff validated only for their own population.

\section{Conclusion}

All the screening tools for sarcopenia performed well to identify non-cases of sarcopenia, with a variable magnitude according to the specific instrument. The promotion of the use of these tools in clinical practice would allow early and targeted management of sarcopenia. Additional study is still needed to develop new and more optimal screening strategies or to determine which tool proves to be more robust in predicting sarcopenia.

\section{Acknowledgments}

This study did not receive any specific grant from funding agencies in the public, commercial or not-for-profit sectors. ML and CB are each supported by a fellowship from the FNRS (National Funding of Research Science, Belgium).

\section{Disclosure}

The authors report no conflicts of interest in this work.

\section{References}

1. Grimby G, Saltin B. The ageing muscle. Clin Physiol. 1983;3(3):209-218.

2. Goodpaster BH, Park SW, Harris TB, et al. The loss of skeletal muscle strength, mass, and quality in older adults: the health, aging and body composition study. J Gerontol A Biol Sci Med Sci. 2006;61(10): 1059-1064.

3. Doherty TJ. Invited review: aging and sarcopenia. J Appl Physiol. 2003;95(4):1717-1727.

4. Beaudart C, Zaaria M, Pasleau F, Reginster J-Y, Bruyère O. Health outcomes of sarcopenia: a systematic review and meta-analysis. PLoS One. 2017;12(1):e0169548.
5. Beaudart C, Reginster JY, Petermans J, et al. Quality of life and physical components linked to sarcopenia: the SarcoPhAge study. Exp Gerontol. 2015;69:103-110.

6. Janssen I, Shepard DS, Katzmarzyk PT, Roubenoff R. The healthcare costs of sarcopenia in the United States. J Am Geriatr Soc. 2004;52(1): 80-85.

7. Shafiee G, Keshtkar A, Soltani A, Ahadi Z, Larijani B, Heshmat R. Prevalence of sarcopenia in the world: a systematic review and metaanalysis of general population studies. J Diabetes Metab Disord. 2017;16(1):21.

8. Ethgen $\mathrm{O}$, Beaudart $\mathrm{C}$, Buckinx F, Bruyère $\mathrm{O}$, Reginster JY. The future prevalence of sarcopenia in Europe: a claim for public health action. Calcif Tissue Int. 2017;100(3):229-234.

9. Marzetti E, Calvani R, Tosato M, et al. Sarcopenia: an overview. Aging Clin Exp Res. 2017;29(1):11-17.

10. Beaudart C, Reginster J-Y, Slomian J, Buckinx F, Locquet M, Bruyère O. Prevalence of sarcopenia: the impact of different diagnostic cut-off limits. J Musculoskelet Neuronal Interact. 2014;14(4):425-431.

11. Beaudart C, Reginster JY, Slomian J, et al. Estimation of sarcopenia prevalence using various assessment tools. Exp Gerontol. 2015;61: 31-37.

12. Dupuy C, Lauwers-Cances V, Guyonnet S, et al. Searching for a relevant definition of sarcopenia: results from the cross-sectional EPIDOS study. J Cachexia Sarcopenia Muscle. 2015;6(2):144-154.

13. Guglielmi G, Ponti F, Agostini M, Amadori M, Battista G, Bazzocchi A. The role of DXA in sarcopenia. Aging Clin Exp Res. 2016;28(6): 1047-1060.

14. Denison HJ, Cooper C, Sayer AA, Robinson SM. Prevention and optimal management of sarcopenia: a review of combined exercise and nutrition interventions to improve muscle outcomes in older people. Clin Interv Aging. 2015;10:859-869.

15. Yu SCY, Khow KSF, Jadczak AD, Visvanathan R. Clinical screening tools for sarcopenia and its management. Curr Gerontol Geriatr Res. 2016;2016:1-10.

16. Roberts HC, Denison HJ, Martin HJ, et al. A review of the measurement of grip strength in clinical and epidemiological studies: towards a standardised approach. Age Ageing. 2011;40(4):423-429.

17. Guralnik JM, Ferrucci L, Pieper CF, et al. Lower extremity function and subsequent disability: consistency across studies, predictive models, and value of gait speed alone compared with the short physical performance battery. J Gerontol A Biol Sci Med Sci. 2000;55(4):M221-M231.

18. Cruz-Jentoft AJ, Baeyens JP, Bauer JM, et al. Sarcopenia: European consensus on definition and diagnosis: report of the European Working Group on Sarcopenia in Older People. Age Ageing. 2010;39(4):412-423.

19. Fielding RA, Vellas B, Evans WJ, et al. Sarcopenia: an undiagnosed condition in older adults. current consensus definition: prevalence, etiology, and consequences. International Working Group on Sarcopenia. J Am Med Dir Assoc. 2011;12(4):249-256.

20. Morley JE, Abbatecola AM, Argiles JM, et al. Sarcopenia with limited mobility: an International Consensus. J Am Med Dir Assoc. 2011;12(6):403-409.

21. Chen L-K, Liu L-K, Woo J, et al. Sarcopenia in Asia: consensus Report of the Asian Working Group for Sarcopenia. J Am Med Dir Assoc. 2014;15(2):95-101.

22. Studenski SA, Peters KW, Alley DE, et al. The FNIH Sarcopenia Project: rationale, study description, conference recommendations, and final estimates. J Gerontol A Biol Sci Med Sci. 2014;69(5):547-558.

23. Malmstrom TK, Morley JE, Haren MT, et al. SARC-F: a simple questionnaire to rapidly diagnose sarcopenia. J Am Med Dir Assoc. 2013;14(8):531-532.

24. Goodman MJ, Ghate SR, Mavros P, et al. Development of a practical screening tool to predict low muscle mass using NHANES 1999-2004. J Cachexia Sarcopenia Muscle. 2013;4(3):187-197.

25. Ishii S, Tanaka T, Shibasaki K, et al. Development of a simple screening test for sarcopenia in older adults. Geriatr Gerontol Int. 2014;14(suppl 1): 93-101. 
26. Yu S, Appleton S, Chapman I, et al. An anthropometric prediction equation for appendicular skeletal muscle mass in combination with a measure of muscle function to screen for sarcopenia in primary and aged care. J Am Med Dir Assoc. 2015;16(1):25-30.

27. Rolland Y, Lauwers-Cances V, Cournot M, et al. Sarcopenia, calf circumference, and physical function of elderly women: a cross-sectional study. J Am Geriatr Soc. 2003;51(8):1120-1124.

28. Pinheiro PA, Carneiro JAO, Coqueiro RS, Pereira R, Fernandes MH. "Chair Stand Test" as simple tool for sarcopenia screening in elderly women. J Nutr Health Aging. 2016;20(1):56-59.

29. Tombaugh TN, McIntyre NJ. The mini-mental state examination: a comprehensive review. J Am Geriatr Soc. 1992;40(9): 922-935.

30. Landis JR, Koch GG. The measurement of observer agreement for categorical data. Biometrics. 1977;33(1):159-174.
31. Grimes DA, Schulz KF. Uses and abuses of screening tests. Lancet. 2002;359(9309):881-884.

32. Park SH, Goo JM, Jo C-H. Receiver operating characteristic (ROC) curve: practical review for radiologists. Korean J Radiol. 2004;5(1):11-18.

33. DeLong ER, DeLong DM, Clarke-Pearson DL. Comparing the areas under two or more correlated receiver operating characteristic curves: a nonparametric approach. Biometrics. 1988;44(3):837.

34. Hajian-Tilaki K. The choice of methods in determining the optimal cut-off value for quantitative diagnostic test evaluation. Stat Methods Med Res. Epub 2017 Jan 1:doi: 10.1177/0962280216680383.

35. Reginster J-Y, Cooper C, Rizzoli R, et al. Recommendations for the conduct of clinical trials for drugs to treat or prevent sarcopenia. Aging Clin Exp Res. 2016;28(1):47-58.

36. Naseeb MA, Volpe SL. Protein and exercise in the prevention of sarcopenia and aging. Nutr Res. 2017;40:1-20.
Clinical Epidemiology

\section{Publish your work in this journal}

Clinical Epidemiology is an international, peer-reviewed, open access, online journal focusing on disease and drug epidemiology, identification of risk factors and screening procedures to develop optimal preventative initiatives and programs. Specific topics include: diagnosis, prognosis, treatment, screening, prevention, risk factor modification,

Submit your manuscript here: https://www.dovepress.com/clinical-epidemiology-journal

\section{Dovepress}

systematic reviews, risk and safety of medical interventions, epidemiology and biostatistical methods, and evaluation of guidelines, translational medicine, health policies and economic evaluations. The manuscript management system is completely online and includes a very quick and fair peer-review system, which is all easy to use. 